\title{
Absence of seasonal variation in concentrations of the house dust mite allergen Der p1 in South Manchester homes
}

\author{
S Kalra, P Crank, J Hepworth, C A C Pickering, A A Woodcock
}

\begin{abstract}
Background House dust mite numbers and the concentration of its main allergen, Der p1, depend on ambient temperature and humidity and have been reported to show a seasonal variation in homes in the United States. A study was designed to find out whether Der p1 concentrations vary with the seasons in dust collected from homes in north west England.
\end{abstract}

Methods The concentration of Der p1 was measured in dust, collected every three months from April 1990 to April 1991, from mattresses and from bedroom and living room carpets in 40 houses in the south Manchester area. Twenty four hour recordings of indoor relative humidity were made in 20 houses during the sampling day.

Results Mean concentrations of Der p1 from all three sources rose two to three fold in October. This was in contrast to the 1000 fold variation in concentrations of Der p1 present between houses within each season's sample and less than the 10 fold change considered to be of clinical importance. The autumn increase was paralleled by a rise in humidity. There was no statistical correlation, however, between Der p1 concentrations and relative humidity, house type, ventilation, or double glazing.

Conclusions The results suggest that seasonal variations in exposure to Der p1 exposure in north west England are small and unlikely to be of major clinical importance. The temperature and humidity data showed that the indoor environment remained relatively constant and conducive to mite growth throughout the year.

North West Lung

Centre

S Kalra

C A C Pickering

A A Woodcock

Department of

Microbiology

J Hepworth

Wythenshawe

Hospital,

Manchester M23 9LT

Reprint requests to:

Dr S Kalra

Received 28 October 199

Returned to authors

17 January 1992

Revised version received

26 February 1992

Accepted 21 April 1992 numbers, is the important factor in the production of symptoms. ${ }^{3}$

Seasonal variations in mite populations have been found in Virginia, United States, with increased numbers from June to August, coinciding with the hot and humid summer. The mite breeds in warm, humid environments and requires a minimum temperature of $15^{\circ} \mathrm{C}$ and relative humidity of at least $60 \% .^{5}$ The accompanying increase in Der pl, however, outlasts this increase in mite numbers and concentrations remain high into the winter for several months after mite numbers have fallen, suggesting that the allergen is stable and relatively persistent.

No data for seasonal variability in Der pl concentrations exist for Britain or any other temperate climate. We studied the influence of the seasons on Der pl concentrations in the dust collected from 40 homes in north west England over 15 months. We also examined the link between allergen concentrations and the pattern of hypersensitivity as determined by skin prick testing.

\section{Methods}

We measured Der pl in the dust collected from the homes of 40 patients with atopic asthma, who were selected from the records of the lung function laboratory at Wythenshawe Hospital, Manchester.

SELECTION OF PATIENTS

Patients were selected from a random starting point in the skin test records for 1989. Forty consecutive patients who satisfied the following criteria were recruited. They had to have asthma, be atopic according to skinprick tests, and live within 10 miles of the hospital. No patient who satisfied these criteria refused to participate. The purpose of the study was explained to them-to measure concentrations of allergens in their homes-and they were asked to make no change in their usual cleaning methods.

Atopy was considered present if skinprick testing produced a weal of over $3 \mathrm{~mm}$ with one or more of the following reagents (Allergopharma, Germany): house dust extract, $D$ pteronyssinus, $D$ farinae, cat dander, grass pollen, and tree pollen. Patients were considered to be predominantly sensitive to house dust mite if the reaction to mite extracts ( $D$ pteronyssinus or $D$ farinae) was at least twice as large as to any of the others.

DUST COLLECTION AND DER PI ASSAY

Dust was sampled for Der pl on five occasions at three month intervals over one year, to cover the four seasons (April, July, and 
Geometric means (95\% confidence intervals) of Der p1 concentrations and mean (SE) indoor and outdoor relative humidity at three month intervals from April 1990 to April 1991

\begin{tabular}{|c|c|c|c|c|c|}
\hline & April 1990 & July 1990 & October 1990 & January 1991 & April 1991 \\
\hline Living room carpet & $\begin{array}{l}1669 \\
(1000-3162)\end{array}$ & $\begin{array}{l}1126 \\
(758-1698)\end{array}$ & $\begin{array}{l}2630 \\
(1412-4898)\end{array}$ & $\begin{array}{l}2188 \\
(1479-3236)\end{array}$ & $\begin{array}{l}1786 \\
(1164-2742)\end{array}$ \\
\hline Bedroom carpet & $\begin{array}{l}1659 \\
(1023-3388)\end{array}$ & $\begin{array}{l}1122 \\
(832-1738)\end{array}$ & $\begin{array}{l}2884 \\
(1778-4898)\end{array}$ & $\begin{array}{l}1820 \\
(1096-3020)\end{array}$ & $\begin{array}{l}1138 \\
(802-1611)\end{array}$ \\
\hline Mattress & $\begin{array}{l}2290 \\
(1349-4677)\end{array}$ & $\begin{array}{l}1412 \\
(1096-2188)\end{array}$ & $\begin{array}{l}4073 \\
(2188-7079)\end{array}$ & $\begin{array}{l}2399 \\
(1698-3388)\end{array}$ & $\begin{array}{l}2786 \\
(1854-4198)\end{array}$ \\
\hline Indoor relative humidity ( $\%$ ) & $63 \cdot 7(1 \cdot 3)$ & $68 \cdot 5(1 \cdot 1)$ & $79 \cdot 2(3 \cdot 4)$ & $58.9(3.3)$ & $53.2(1.8)$ \\
\hline Outdoor relative humidity ( $\%$ ) & $81 \cdot 9(1 \cdot 2)$ & $75 \cdot 9(2 \cdot 2)$ & $92 \cdot 1(1 \cdot 0)$ & $90 \cdot 6(1 \cdot 1)$ & $73 \cdot 6(1 \cdot 2)$ \\
\hline
\end{tabular}

*There was a significant $(p<0.05)$ increase in Der pl concentrations in mattresses and bedroom carpets and in indoor relative humidity in October 1990.

October 1990; January, April 1991). The collection was made in a standardised manner by vacuuming for five minutes with a Medivac vacuum cleaner (Taylormaid, Macclesfield) on to preweighed filter paper (Whatman GFF, pore size $0.7 \mu \mathrm{m})$. Samples were taken from $2 \mathrm{~m}^{2}$ of bedroom and living room carpet, and from $1 \mathrm{~m}^{2}$ of the upper surface at the head end of each patient's mattress; the same sites were sampled at each visit. Samples were coded and stored at $4^{\circ} \mathrm{C}$ until analysis. The dust was weighed after removing obvious "non-dust" matter. Der p1 was extracted $(1: 5 \mathrm{w} / \mathrm{v})$ overnight at room temperature into phosphate buffered saline containing $0.05 \%$ Tween 20 and $0.2 \%$ bovine serum albumin. Samples were then vigorously homogenised and centrifuged at $3000 \mathrm{rev} / \mathrm{min}$ for 15 minutes and all extracts were stored at $-20^{\circ} \mathrm{C}$ until analysis. Der pl was assayed blind, in duplicate, by an enzyme linked immunosorbent assay (ELISA) technique (ALK Laboratories, Copenhagen) and was expressed in $\mathrm{ng} / \mathrm{g}$ crude dust. Calibration curves were constructed with the manufacturer's reference samples and the spectrophotometer software was programmed to reject paired samples if they differed by more than $5 \%$. This restricted intra-assay variation to this level. Batch to batch (interassay) variation was assessed by repeated assays of multiple house dust samples and was consistently $13-15 \%$, less

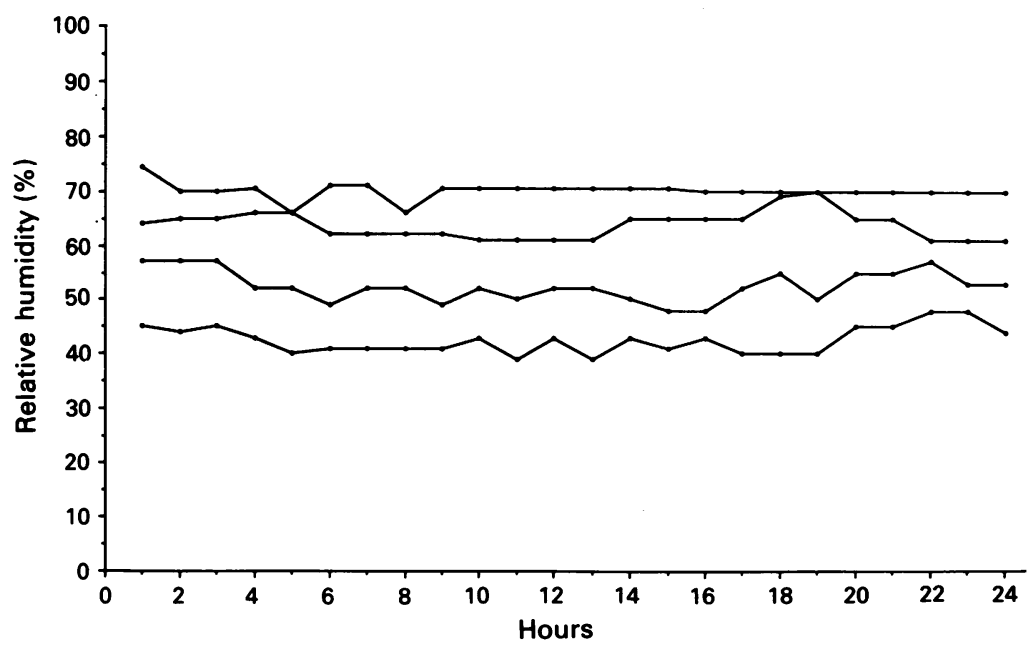

Figure 1 Four sample traces from four different houses of hourly humidity measurements on dust sampling days to show the stability of the indoor environment. The samples were chosen to illustrate the constancy at four different levels of relative humidity. Analysis of serial measurements made in 20 houses showed no significant hour to hour variation. than the manufacturer's figure of $25 \%$ for crude dust Der p1.

\section{INDOOR ENVIRONMENT}

Details of house type, ventilation, heating, and location in relation to open water (rivers, lakes, etc) were recorded. At the first visit, in April 1990, a single measurement of dry and wet temperature was made at the time of sampling and relative humidity was calculated with a standard nomogram. At subsequent visits, in 20 houses selected randomly, we used a data logging device (Squirrel, Grant Instruments, Cambridge) to make hourly recordings from dry and wet temperature probes over 24 hours on the sampling day. The hourly relative humidity was calculated from these recordings and mean concentrations for each 24 hours were used for analysis. All readings were taken in the bedroom that was sampled for allergen.

\section{ANALYSIS}

The Der pl concentrations were log transformed and concentrations at each collection time point were compared with each other by using repeated measures of analysis of variance. The changes in relative humidity were analysed with the same test. Relationships between humidity and Der pl concentrations were assessed by linear regression and correlation coefficients. Significance was set at the conventional $5 \%$ level.

\section{Results}

Of the 40 houses studied, $38(95 \%)$ were centrally heated and $15(37.5 \%)$ had double glazing. This reflected the general pattern of housing and was consistent with other data, which show that over $90 \%$ of houses in Greater Manchester are centrally heated (personal communication, Manchester City Council).

Analysis of the hourly humidity measurements from all 20 houses (with repeated measures of analysis of variance) recorded at each sampling time did not show any significant hour to hour variation. Figure 1 shows the hourly concentrations recorded in four of the houses to illustrate this indoor stability in houses of different levels of humidity. On this basis we assumed that average and spot readings were comparable and therefore all humidity data, however obtained, were pooled together for analysis. Mean concentrations are 

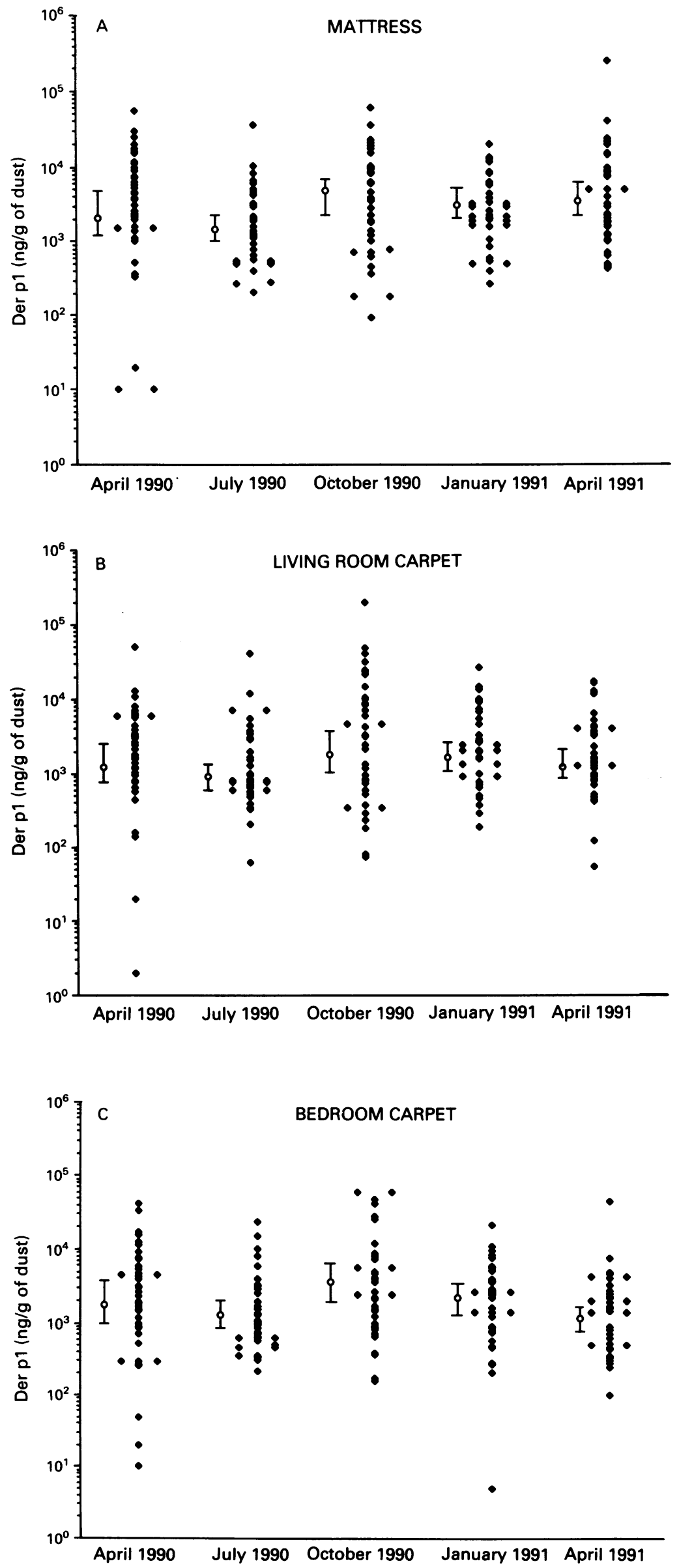

Figure 2 Der $p 1$ concentrations in $(A)$ mattresses, $(B)$ living room carpets, and $(C)$ bedroom carpets from April 1990 to April 1991 . Error bars indicate $95 \%$ confidence intervals (see table for mean values). The increase in October was significant, but small, in mattresses and bedroom carpets. shown in the table along with mean outdoor humidity for the same periods.

The table also shows the mean concentrations of Der pl in each of the three sampling sites-that is, living room and bedroom carpets and mattresses. There was an increase in the mean Der p1 concentrations in bedroom carpets and mattresses $(p<0.05)$ and living room carpets (not significant) from the low in July to the high in October. The increase seen in October was the result of an increase in Der p1 to its highest level during the study in 15 mattresses, 14 bedroom carpets, and 15 living room carpets. There was no significant difference between the concentrations measured at any of the other sampling points.

The weight of collected dust was similar from the three sites. The mean (SE) of all samples taken from each area was: mattresses $0.85(0.05) \mathrm{g}$, bedroom carpet $1.11(0.05) \mathrm{g}$, and living room carpet $0.99(0.06) \mathrm{g}$. The weight of dust also did not vary significantly between sampling points, though slightly lower concentrations were seen in October than at the other four points. When total allergen loads (expressed as the product of dust weight and Der p1 concentration) were compared there were no significant differences between any of the five sets of samples. As has been emphasised in earlier reports ${ }^{3}$, however, the dust collection process is the most variable step of the assay and therefore the preferred comparison is between allergen concentrations rather than total content.

Although statistically significant, the magnitude of change in Der $p 1$ concentrations (twofold to threefold) was small by comparison with the wide variation seen between the houses at each sampling point. This variation, which ranged from 10 to $250000 \mathrm{ng}$ Der p1/g of dust, is seen in figure 2 , which shows the concentrations found at each of the sampling sites in the 40 houses.

This wide inter-house variation contrasts with a small intra-house variation, the variation between sampling sites being of the same twofold to threefold order as the overall change through the seasons. The mean coefficients of variance $(95 \%$ confidence interval) of $\log$ normalised Der $\mathrm{p} 1$ values from each sampling site from each house over the study period were: living room carpet $16.9 \%(14.3-19.5 \%)$; bedroom carpet $15 \cdot 9 \%(13 \cdot 3-18 \cdot 4 \%)$; mattresses $16 \cdot 8 \%(14 \cdot 1-19 \cdot 5 \%)$.

Accompanying the rise in Der p1 concentrations in October 1990 was the highest mean relative humidity recorded at any of the five sampling times (table) but there was no correlation between humidity and Der p1 concentrations in dust from any site. There was also no correlation between the increase in humidity and the increase in allergen concentrations between the lowest point (July) and the highest point (October). In all but four houses relative humidity readings were above $50 \%$ throughout.

Seventeen patients were sensitive only or predominantly to $D$ pteronyssinus; the remaining 23 showed mixed reactions, all having positive skinprick responses to pollens and one 
or more of the other agents. There was no difference between these two groups in the Der p1 concentration in the dust from any of the three sampling sites in their homes.

\section{Discussion}

Few data are available on the seasonal variation in Der $\mathrm{pl}$ concentrations in temperate regions. This study was primarily designed to provide data on seasonal variations in Der p1 concentrations in houses in South Manchester and therefore a random sample of patients was used. This reflects the general pattern of housing in the area. No attempt was made to pick a sample stratified according to house design or type of heating. Therefore this study reflects Der p1 levels in predominantly centrally heated, well insulated houses, which are highly representative of housing in the United Kingdom.

We found only a small change in concentrations of mite antigen with the seasons. This two to three fold change was considerably less than the 100-1000 fold change reported by Platts-Mills ${ }^{5}$ from Virginia. Changes of clinical significance are likely to be of a greater magnitude (10 fold or more). ${ }^{3}$ The smaller increase in autumn in the houses we studied therefore, though statistically significant, is unlikely to be of major clinical importance. This is supported by the absence of any evidence of an increase in the incidence of asthma exacerbations in the autumn. ${ }^{6}$

We found a much larger (1000-10 000 fold) variation between houses at each sampling point. This greatly overshadows the two to three fold increases between seasons and further diminishes their importance. The determinants of this variation are likely to be complex and probably include an interplay of house related factors, such as design, location (especially in relation to open water), age, and type and age of soft furnishings, as well as long term indoor environmental conditions, including temperature, humidity, and ventilation. Within this variation, however, houses tended to be consistent, high concentrations of Der pl remaining high through the year and low concentrations remaining low.

Temperatures above $15^{\circ} \mathrm{C}$ and, more importantly, relative humidity above $50 \%$ are conducive to mite breeding, ${ }^{4}$ and these conditions were present throughout the year in nearly all the houses we studied. Only four houses had relative humidity below $50 \%$ during the study period. These constant indoor conditions are the result of modern house design, notably the near universal use of central heating, and favour mite breeding throughout the year. The lack of correlation between humidity and allergen concentrations suggests that, once minimum conditions for mite breeding are satisfied, mite growth occurs independently of this factor.

It has been suggested that people with house dust mite sensitive asthma are exposed to higher concentrations of allergen. ${ }^{7}$ We found no association between high domestic Der p1 concentrations and a positive skinprick test response in our 40 patients, of whom 17 were predominantly house dust mite sensitive. If exposure to Der p1 in early life is the important factor determining hypersensitivity, the lack of correlation is not surprising. Current exposure to high concentrations is probably more important in relation to asthma symptoms, but this is an issue we did not examine in this study.

In conclusion, we found that Der $\mathrm{p} 1$ concentrations in the houses we examined remained relatively constant throughout the year, though there was a small increase in October, which was accompanied by an increase in indoor relative humidity. The constant indoor environment favours mite breeding and changes in house design may be necessary to make homes less ideal for this producer of potent allergens.

1 Voorhorst R, Spieksma FThM, Varekamp H, Leupen MJ, Lyklema AW. The house dust mite (Dermatophagoides pteronyssinus) and the allergens it produces: identity with the house dust allergen. $J$ Allergy 1967;39:325-39.

2 Sibbald B, Rink E, D'Souza M. Is the prevalence of atopy increasing? Br J Gen Pract 1990;40:338-40.

3 Anonymous. Report of an international workshop. Dust mite allergens and asthma-a worldwide problem. $J$ Allergy Clin Immunol 1989;83:416-27.

4 Platts-Mills TAE, Hayden ML, Chapman MD, Wilkins SR. Seasonal variation in dust mite and grass-pollen allergens in dust from the houses of patients with asthma. $J$ Allergy Clin Immunol 1987;79:781-91.

5 Colloff MJ. Differences in development time, mortality and water loss between eggs from laboratory and wild populations of Dermatophagoides pteronyssinus. Experimental and Applied Acarology 1987;3:191-200.

6 Murray AB, Ferguson AC, Morrison B. The seasonal variation of allergic respiratory symptoms induced by house dust mites. Ann Allergy 1980;45:347-55.

7 Korsgaard J. Mite asthma and residency. A case control study on the impact of exposure to house dust mites in dwellings. Am Rev Respir Dis 1983;128:231-5. 